\title{
The role of the innate immune system in the development and treatment of hepatocellular carcinoma
}

\author{
Christoph Roderburg ${ }^{1}$, Alexander Wree' ${ }^{1}$ Münevver Demir ${ }^{1}$, Moritz Schmelzle² \& Frank \\ Tacke*, 1 iD \\ ${ }^{1}$ Department of Hepatology \& Gastroenterology, Charité, Universitätsmedizin Berlin, Humboldt-Universität zu Berlin, Campus \\ Charite Mitte, Campus Virchow-Klinikum, Berlin Institute of Health, Augustenburger, Platz 1 13353, Berlin \\ ${ }^{2}$ Department of Surgery, Charité, Universitätsmedizin Berlin, Humboldt-Universität zu Berlin, Campus Charité Mitte, Campus \\ Virchow-Klinikum, Berlin Institute of Health, Augustenburger, Platz 1 13353, Berlin \\ *Author for correspondence: frank.tacke@charite.de
}

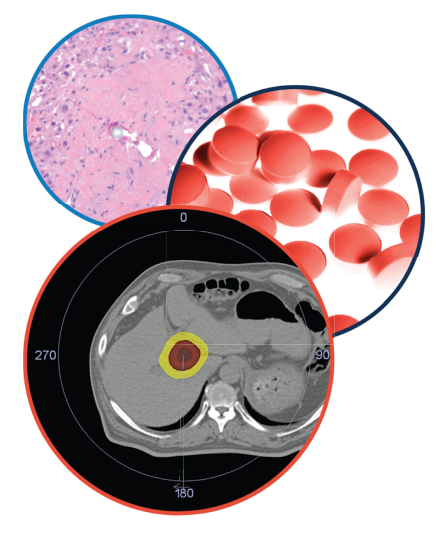

Hepatic Oncology

Hepatocellular carcinoma (HCC) is the most common primary liver cancer. Most patients present with advanced or metastatic HCC at diagnosis and face a dismal prognosis. Tyrosine kinases are the gold standard treatment for this disease but yield limited survival benefits. Immune checkpoint inhibitors that augment adaptive immunity have been tested in HCC. Complex interactions between tumor cells, lymphocytes and the tumor environment determine the efficacy of such immunotherapies. Innate immune mechanisms known drivers of liver disease progression in pre-HCC conditions such as fibrosis or cirrhosis - may either support or counteract tumor-related immune activation. In this review, we will highlight current concepts of the role of the innate immune system in hepatocarcinogenesis and discuss their relevance for translation into clinics.

First draft submitted: 12 August 2019; Accepted for publication: 15 January 2020; Published online: 31 January 2020

Keywords: checkpoint inhibition $\bullet$ HCC $\bullet$ hepatocellular carcinoma $\bullet$ immunotherapy $\bullet$ innate immune system • macrophages

Hepatocellular carcinoma (HCC) is one of the most lethal cancers worldwide. In previous decades both incidence and mortality of liver cancer dramatically increased in industrialized countries, including Europe, North and South America [1]. In up to $90 \%$ of cases, HCC arises in cirrhotic or chronically inflamed livers. HCC is considered as the most prominent life-limiting complication of liver cirrhosis [2]. Liver cirrhosis is the common end-stage of chronic liver diseases, such as hepatitis $\mathrm{B}$ or $\mathrm{C}$ virus infection, alcohol abuse and nonalcoholic fatty liver disease (NAFLD) [3]. Due to the epidemic spread of metabolic diseases, liver cancer is among the fastest growing causes of cancer-related death. A model-based simulation forecasts continued increases in HCC mortality until 2030 underscoring the tremendous relevance of this disease [4].

Despite intensive research activities, most patients with HCC still face a dismal prognosis. Only a minority of patients with early HCC can be scheduled for liver transplantation; patients undergoing alternative curative procedures - such as liver resection or tumor ablation - develop recurrent disease in up to $70 \%$ of cases [5]. Patients who are not considered suitable for surgical or interventional strategies, for example, due to advanced tumor stages, can be offered palliative drug therapies including tyrosin kinase inhibitors (TKI) such as sorafenib, lenvatinib, regorafenib or cabozantinib, the VEGFR2-antibody ramucirumab or best supportive care [6-9]. However, these substances yield only marginal survival benefits and are regarded as not cost-effective [10], highlighting the need for novel therapeutic strategies for treatment of HCC.

Chronic inflammation has been shown to promote development of various types of cancer [11,12]. Intensive research has been performed to decipher the immunological mechanisms that are involved in initiation and progression of liver cancer (Table 1). Just recently, immunotherapies have been introduced into clinical treatment algorithms of many cancers such as malignant melanoma, lung cancer and hematologic cancers [13,14], including HCC $[15,16]$. Intensive research activities have deciphered the immunological mechanisms that are involved in the 
Table 1. Role of different innate immune components in hepatocellular carcinoma.

\begin{tabular}{|c|c|}
\hline Immune component & Function(s) in HCC \\
\hline $\mathrm{KC}$ & $\begin{array}{l}\text { Promote immunosuppression and hepatocarcinogenesis } \\
\text { Correlate with tumor progression in humans }\end{array}$ \\
\hline DC & $\begin{array}{l}\text { Act as messengers between the innate and the adaptive immune systems } \\
\text { Promote immunotolerance } \\
\text { Association to initiation and progression of HCC } \\
\text { Indirectly promote proliferation of transformed hepatocytes through inhibitory effects on } \mathrm{CD}^{+} \mathrm{T} \text { cells } \\
\text { Increased numbers were found in human HCC }\end{array}$ \\
\hline Myeloid-derived suppressor cells & $\begin{array}{l}\text { Suppress both the arms of immune system: innate and adaptive immunity } \\
\text { Induced from monocytes/macrophages by differentiation signals from the tumor environment and stellate } \\
\text { cells } \\
\text { Support tumor growth } \\
\text { Promote the expansion of Tregs } \\
\text { Promote the conversion of } \mathrm{CD}^{+} \mathrm{T} \text { cells into Tr1 cells }\end{array}$ \\
\hline Neutrophils & $\begin{array}{l}\text { Promote hepatocarcinogenesis } \\
\text { Interact with } \mathrm{KCs} \text { and recruit Tregs as well as macrophages to promote immune tolerance } \\
\text { Promote resistance to sorafenib }\end{array}$ \\
\hline NK cells & $\begin{array}{l}\text { Antifibrotic activity through targeting activated HSCS } \\
\text { Diminished function during the development of HCC } \\
\text { Promote angiogenesis through MMP9, cytotoxic activity } \\
\text { Reduced amounts of NK cells were found in HCC lesions, and the number of CD56 } 6^{+} \text {NK cells was correlated } \\
\text { with the prognosis in HCC patients }\end{array}$ \\
\hline NKT cells & $\begin{array}{l}\text { Directly activate HSCS } \\
\text { Prevent progression of } \beta \text {-catenin-driven HCC } \\
\text { Involved in the immune surveillance of senescent hepatocytes } \\
\text { Increased number in human NASH, cirrhosis and HCC }\end{array}$ \\
\hline
\end{tabular}

initiation and progression of liver cancer, highlighting the potential of these therapies to treat cancer [17]. In the context of HCC, various studies on the effects of immunotherapies have been conducted with partially conflicting results $[18,19]$, which might be explained by the fact that the efficacy of immunotherapies depends on very complex and only poorly understood interactions between many different immune cells, tumor cells and cells of the tumor environment.

Many of the immune cells infiltrating solid tumors or the tumor microenvironment belong to the innate arm of the immune system [20]. Innovative methodologies such as single-cell RNA sequencing technologies revealed a strikingly heterogeneous composition of immune cells in human liver cirrhosis and HCC, particularly manifold macrophage and dendritic cell (DC) populations, whose distinct functions are only partially understood at present $[22,23]$. These exciting data fuel the expectation that modulating innate immune cells to improve available immunotherapies or as an alternative immunotherapeutic option might help to overcome limitations of current immunotherapeutic approaches (which largely rely on the adaptive arm of the immune system) in the context of HCC [24]. However, the functionality of innate cells is regulated by several factors, including pro- and anti-inflammatory cytokines as well as fibroblasts, endothelial cells and many other cell types within the tumor or the tumor environment [25]. The complexity of this regulation makes a fast translation of such concepts into clinical routine challenging. In this review, we summarize available data on the role of innate immune responses in hepatocarcinogenesis and discuss its prognostic implications as well as its emerging role as a therapeutic target for HCC.

\section{Current immunotherapeutic approaches for HCC}

Numerous immunological approaches have been investigated in the recent past to prevent the development of tumors in cirrhotic livers or to control tumors that have already developed (Table 2). However, the results of immunotherapeutic approaches in HCC have remained, for a long time, largely disappointing. As an example, vaccination strategies have not shown significant benefit, and broad anti-inflammatory treatments did not reduce HCC rates in cirrhotic livers in Phase III trials [26]. In recent years, immune checkpoint blockade been introduced into therapeutic algorithms of many different cancers [27]. Modulation of checkpoint receptors such as PD1 or CTLA-4 might reinstate host immune response against malignant tumors, providing tumor control [27]. Both the CheckMate-040 trial and the KEYNOTE-224 trial have reported promising results for the anti-PD-1 antibodies nivolumab and pembrolizumab after failure of TKI therapy in patients with advanced or metastatic 
Table 2. Immunotherapeutic strategies to treat hepatocellular carcinoma.

\begin{tabular}{|ll|}
\hline Immunotherapy & Application details \\
\hline Vaccines & $\begin{array}{l}\text { Antibodies (pembrolizumab Ipilimumab, } \\
\text { nivolumab, atezolizumab and others) }\end{array}$ \\
\hline Antigenic peptides/proteins
\end{tabular}

Mode of action

Inhibition of specific receptors and receptor ligands (PD-1, PD-L1, CTLA-4 and others) to booster antitumor immune responses

Tumor-associated antigens are targeted to overcome immune tolerance

Transfer of tumor-specific $\mathrm{T}$ cells from a healthy individual into HCC patients

Patient-derived T cells are modified ex vivo and retransferred into the donor (HCC patient)

HCC: Hepatocellular carcinoma.

HCC $[18,19]$. Despite two large Phase III trials failure to confirm results from these earlier trials, the KEYNOTE240 trial (pembrolizumab does not significantly improve survival in patients with pretreated HCC) and the CheckMate-459 trial (nivolumab does not improve overall survival compared with sorafenib in patients with newly diagnosed unresectable HCC), recent results from the IMBRAVE-150 are widely considered as breakthrough for immunotherapy in HCC. Within this study, 501 patients with advanced HCC without prior systemic therapy were randomized to the experimental arm (the anti-PD-1 atezolizumab plus the VEGF inhibitor bevacizumab) or the control arm (sorafenib). The combination therapy significantly improved the overall survival of advanced HCC patients. In the combination arm, the 6-month survival rate was $85 \%$ compared with $72 \%$ in the sorafenib arm, median survival time was not even achieved in the combination arm so far, while it was 13.2 months in the sorafenib arm (hazard ratio [HR]: 0.55). Similarly, progression-free survival of patients under immunotherapy was significantly better than under sorafenib (6.8 vs 4.3 months; HR: 0.59$)$. With less toxicity, the quality of life of patients on atezolizumab plus bevacizumab was significantly improved and the authors concluded that 'atezolizumab + bevacizumab should be considered a practice-changing treatment for patients with unresectable HCC who have not received prior systemic therapy,' highlighting the tremendous potential of immune therapy in patients with HCC.

Nevertheless, even with atezolizumab and bevacizumab, response rates are rather low. At present, the question remains unsolved why checkpoint inhibition does only provide moderate effects in HCC, an inflammation-driven tumor, when compared with other malignancies. However, answers to this question could potentially improve immunotherapy in HCC. Mechanisms potentially mediating resistance against checkpoint inhibition in the setting of HCC might include (but are not limited to) induction of T-cell anergy, inhibition of effector T-cell migration, T-cell inactivation via specific receptor-ligand interactions and barrier functions of the stroma [28-30]. Moreover, macrophages, neutrophils and other immune cells of the innate arm were suggested to be involved in mediating resistance against checkpoint inhibitors [31]. Just recently, a role for tumor-associated neutrophils in mediating resistance against sorafenib was described [32]. In line, Cheng et al. suggested that activated PD-L1 ${ }^{+}$neutrophils might exert a protumor effect by suppressing T-cell immunity in a PD1/PD-L1-dependent manner within the tumor microenvironment, highlighting the role of the innate arm of the immune systems in mediating response to $\mathrm{PD}(\mathrm{L})-1$ directed therapies in HCC [33]. Besides neutrophils, recent results indicated an important role of natural killer (NK) cells, which express immunological checkpoint molecules such as PD-1 and CTLA-4 to a significant extent. PD-1/PD-L1 blockade has been shown to augment NK cell-mediated tumor lysis in multiple myeloma. Moreover, it was suggested that targeting PD-1/PD-L1 might also activate NK cells [34]. Besides PD-1 and CTLA-4, TIM-3 and LAG-3 represent potential NK cell immune checkpoints (summarized in [35]). Both have emerged as targets for cancer immunotherapy, due to their capacity of negatively regulating T-cell activation and synergizing with PD-1 to exhaust $T$ cells [36]. At present, several ongoing clinical trials are exploring the therapeutic efficacy of LAG-3 and PD-1 combined treatment with various advanced cancers [35]. Thus, cells of the innate arm of the immune systems might not only affect efficacy of current immunotherapies but are also represent targets for novel immunotherapeutic approaches in cancer.

\section{Role of innate immune cells for hepatocarcinogenesis \& immunotherapy}

In recent years, tumor-infiltrating immune cells have been intensively analyzed and characterized (Table 1). For numerous solid tumors, including primary liver cancer, associations between certain immune cell populations and response to therapy as well as on prognosis have been proposed [17,37-39]. Although the precise significance of the tumor immune microenvironment is still not fully understood, a high density of myeloid cells is often associated 
with a poor prognosis and a high density of infiltrating T-effector cells is often associated with a good prognosis [40,41]. Thus, understanding the immune microenvironment may predict, guide and improve immunotherapy [42].

During the progression of liver diseases, inflammation is considered a driving factor and a prerequisite for liver cancer [43]. Some of these 'tumor-promoting' aspects of inflammation in chronic liver diseases include hepatocyte cell death followed by aberrant regeneration, fibrosis or angiogenesis [44]. Technological advances such as single-cell RNA sequencing provide a more in-depth understanding of cellular heterogeneity in the inflamed environment of a fibrotic or cirrhotic liver [45-48]. However, malignant tumors also create an intrinsic inflammatory response, favoring antitumor responses in some of the cases [12,49].

\section{Monocytes \& macrophages}

The hepatic immune response provoked by HCC has been examined in different mouse models and retraced in blood and tissue samples from patients with HCC. For instance, the lack of T- and/or B-cells increases chemically induced liver cancer, supporting that adaptive immune responses limit hepatocarcinogenesis [50]. On the other hand, a prolonged activation of both Kupffer cells (KCs) and inflammatory monocytes represents a characteristic (pathological) feature observed in the context of chronic liver inflammation [51], potentially leading to HCC. In this setting, chemokine CCL2/chemokine receptor CCR2-dependent signaling mechanisms were suggested to be involved in the process of hepatocarcinogenesis. CCL2 is expressed at higher levels in patients with HCC, and elevated CCL2 expression levels were indicative for an impaired prognosis [29]. In murine models of liver cancer, CCR2 ${ }^{+}$myeloid cells exert a context-dependent function: while they have a protective role via clearance of senescent (premalignant) hepatocytes and CCR2 ablation was associated with increased tumor burden, they were demonstrated to have a tumor-promoting role through NK cell inhibition once HCC established [52]. Interestingly, treatment with a CCR2 antagonist inhibited HCC growth and metastasis, ultimately enhancing survival in a model of senescence-associated HCC [52], while in a model of nonalcoholic steatohepatitis (NASH)-dependent HCC, CCR2 depletion had no clear effect on HCC tumorigenesis [53], suggesting that the effect of CCR2 in hepatocarcinogenesis depend on disease etiology. Functionally, blocking of the CCL2/CCR2 axis was associated to an inhibition of the recruitment of inflammatory monocytes, infiltration and repolarization ('M2') of tumorassociated macrophages and an activation of an antitumorous $\mathrm{CD} 8^{+} \mathrm{T}$-cell response [54]. These CCR2-dependent macrophages also promote pathogenic angiogenesis for tumor vascularization in fibrotic livers [55]. The group of Yao et al. extracted a novel CCR2 inhibitor, named 747, from Abeis georgei. Administration of 747 to HCCbearing mice was associated with an increase of intratumoral $\mathrm{CD} 8^{+} \mathrm{T}$ cells leading to an activation of antitumoral immune responses and to tumor control in orthotopic and subcutaneous HCC. Moreover, 747 demonstrated additive effects when used in combination with sorafenib, indicating that the immunomodulators might represent a therapeutic option to improve current treatment strategies in the context of HCC [56]. From a clinical perspective, inhibiting CCL2/CCR2 axis is a realistic therapeutic option in the near future, because the dual chemokine receptor CCR2/CCR5 inhibitor cenicriviroc is under development for patients with NASH and fibrosis [57]. This drug effectively reduces the recruitment of inflammatory monocytes to injured livers, which ameliorates hepatic fibrosis [51,58]. The potential benefits for HCC development are, however, currently unknown.

\section{Kupffer cells}

A role for KCs, the resident macrophages in the liver, in hepatocarcinogenesis was described in the context of chemical carcinogen-induced liver cancer, where they promote carcinogenesis in a complex and yet not fully understood interaction with hepatocytes [59]. Dying hepatocytes might release danger signals ('DAMP') triggering activation of KCs via Toll-like receptors. Moreover, increasing evidence is available for an activation of KCs via upregulation of HIF-1 $\alpha$, occurring in the context of liver hypoxia [60]. Another activating pathway for KCs that appears to be highly relevant during hepatocarcinogenesis is the hyaluronan-CD44-dependent binding of platelets. The therapeutic inhibition of platelet cargo, platelet adhesion and platelet activation resulted in reduced KC activation, improved NASH phenotype and reduced carcinogenesis in mouse models of HCC [61]. KC, but also monocyte-derived macrophages, releases cytokines like TNF that triggers liver cancer by inducing mitochondrial dysfunction and reactive oxygen species accumulation [62].

Deciphering the exact roles of KCs and monocyte-derived macrophages (as a prerequisite for specific therapeutic targeting) is further hampered by the heterogeneity of these myeloid cells regarding origin/ontogeny, activation pathways and functions in health and disease [63]. This has been demonstrated in the highly prevalent conditions of NAFLD and NASH, in which HCC might occur even in the absence of liver cirrhosis. During progression of 
NAFLD upon western diet feeding in mice, single-cell RNA sequencing analyses revealed a unique inflammatory activation profile among different myeloid cell populations in liver and simultaneously in bone marrow, which has implications for injury recognition, metabolism and immune activation [46]. Following the detailed description of macrophages in the liver, many pathways were identified that contribute to the proinflammatory polarization of macrophages during $\mathrm{NASH}$, including changes in the gut microbiota, an altered metabolism that diminish glucocorticoid signaling or epigenetic changes [64,65]. Within macrophages, the formation of inflammasome complexes promotes inflammatory activation, and inhibition of the NLRP3 molecule protected from NASH in rodent models [66]. Intriguingly, activation of inflammasomes provide a link to the development of liver cancer in NASH via production of IL-6, which has a mitogenic effect on hepatocytes and promotes HCC [67]. In addition, activated hepatic macrophages promote NASH and the development of HCC through the production of reactive oxygen species [68-70].

\section{Myeloid-derived suppressor cells}

The functionality of immune cells in the microenvironment of malignant tumors represents a highly regulated process. In the context of HCC, monocyte-derived macrophages were identified to contribute to the recognition and clearance of senescent (premalignant) hepatocytes, which prevents tumor development [52]. In the environment of an established HCC, monocyte-derived cells can acquire a phenotype of myeloid-derived suppressor cells (MDSCs), which support tumor growth [71]. Tumor-induced MDSCs demonstrate positivity for the myeloid surface marker $\mathrm{CD} 11 \mathrm{~b}$ and the granulocyte/monocyte marker Gr1 in mice [72]. Various studies described the hepatic enrichment of $\mathrm{CD} 1 \mathrm{~b}^{+} \mathrm{Gr}^{+}$immune cells in experimental liver cancer and of similar cells in human HCC [71]. MDSCs promote the expansion of Tregs and promote the conversion of $\mathrm{CD}^{+} \mathrm{T}$ cells into Tr 1 cells. In HCC, Tregs mediate tumor-induced immune tolerance and promote immune escape [73]. Besides Tregs, DCs might promote immunotolerance and thereby lead to initiation and progression of HCC [74,75]. Targeting MDSCs, for example, by blocking their T-cell-suppressing activity, could therefore be attractive to enhance the efficacy of immunotherapy. This has been exemplarily demonstrated in mouse models of liver cancer, in which suppressing MDSC enhanced the therapeutic efficacy of 'cytokine-induced killer cells' that were administered to tumor-bearing mice [76].

More recently, several studies addressed interactions between hepatic stellate cells (HSCs) and monocytes/macrophages for understanding mechanisms driving the development of MDSCs. Using coculture systems, mouse models and human samples for validation, HSCs were found to induce MDSC differentiation in the liver tumor environment $[77,78]$. Activated HSCs induced p38 MAPK signaling in macrophages, which promoted their reprogramming toward MDSC development that suppressed (antitumoral) T-cell activity. In turn, interfering with the HSC-MDSC interaction by the p38 MAPK inhibitor reduced HCC growth in preclinical models [78]. These data principally demonstrate that interfering with the innate immune environment of HCC could potentiate immunotherapeutic approaches.

\section{Neutrophils}

Neutrophils represent the most abundant leukocytes in peripheral blood. Moreover, neutrophils are among the most common tumor-infiltrating immune cells. Recent data have suggested that neutrophils play an important role in the regulation of tumor initiation and progression [79,80]. In line, elevated numbers of neutrophils are associated to an impaired prognosis of patients with solid tumors in general [80] and in patients with HCC in particular [81]. Mechanistically, it was demonstrated in different non-HCC tumor entities that neutrophils promote tumor cell proliferation [82], tumor vascularization, migration and invasion [83-85]. Moreover, neutrophils might suppress antitumor immunity [86] by producing a plethora of prooncogenic ligands [87]. Recently, neutrophils were also suggested to promote hepatocarcinogenesis (e.g. [32]). In HCC, tumor-associated neutrophils closely interact with KCs and recruit Tregs as well as macrophages [32], thereby leading to immune tolerance. More specifically, immunohistochemical analysis revealed that $\mathrm{CCL} 2^{+}$and $\mathrm{CCL} 17^{+}$cells, coexpressing CD66b (thereby marking them as neutrophilic cells), were enriched within the HCC microenvironment and correlated with tumor size, microvascular invasion, tumor encapsulation, tumor differentiation and stage. Strikingly, higher levels of these cells indicated an unfavorable prognosis in HCC patients [32]. In mice, tumor-associated neutrophils promoted resistance to sorafenib, while in turn combining sorafenib with a neutrophil-inhibiting agent provided superior tumor control than sorafenib alone [32]. Thus, inhibition of tumor-associated neutrophils might represent a novel therapeutic concept using cells from the innate arm of the immune system. In line to these data, Cheng et al. recently demonstrated that the phenotype and function of neutrophils in HCC is influenced by cancer-associated 
fibroblasts (CAF) [33]: neutrophils were activated by conditioned medium from CAF with increased expression of CD66b, PD-L1, IL-8, TNF- $\alpha$ and CCL2, and with decreased expression of CD62L. CAF-primed neutrophils impaired T-cell function through the PD-1/PD-L1 signaling pathway [33], highlighting a mechanism by which cells from the innate arm of the immune system might affect response to checkpoint inhibition in HCC.

NKT cells

NKT cells represent a subpopulation of lymphocytes that are activated very early in an immune response but lack immunological memory. As such, most authors attribute NKT cells to the innate immune system ('innate lymphocytes') rather than to the adaptive immune system. When activated, NKT cells produce Th1 or Th2 cytokines [30]. Our group has recently shown that iNKT cells are recruited in a CXCR6-dependent manner to the liver already during the very early phase of immune response in different models of liver injury [88]. CXCR6 ${ }^{+}$-NKT cells in turn activate local macrophages, thereby further enhancing inflammation [89]. NKT cells are also involved in antitumor immunity. NKT cells prevent development of tumor metastasis in manifold cancers. Moreover, iNKT cells from patients with malignant diseases secrete less IFN- $\gamma$ compared with tiNKT cells derived from healthy controls [90]. The specific role of NKT cells in hepatocarcinogenesis is less well understood. Just recently, our group demonstrated that CXCR6-deficient mice had a significantly higher tumor burden than wild-type mice in a model of diethylnitrosamine-induced liver cancer in mice. Notably, CXCR6 deficiency was associated with reduced intrahepatic numbers of invariant NKT and $\mathrm{CD}^{+}{ }^{+} \mathrm{T}$ cells that express TNF and IFN- $\gamma$, which was also seen by a peritumoral accumulation of CXCR6-associated lymphocytes in human HCC [91]. On a functional level, NKT but also $\mathrm{CD}^{+} \mathrm{T}$ cells were involved in the immune surveillance of senescent hepatocytes [91]. The hepatic recruitment of $\mathrm{CXCR}^{+}$NKT involves the upregulated expression of its ligand CXCL16 on liver sinusoidal endothelial cells. Interestingly, endothelial CXCL16 expression in the liver is controlled by gut microbiome-mediated primary-tosecondary bile acid conversion, thereby linking gut microbiota to liver antitumor immunosurveillance [92].

Next to these data on HCC, it was reported that NKT promotes liver fibrosis [88,93,94]. Thus, available data point toward a dual role of NKT cells in tumor immunity. While they promote tumor rejection by Th1 cytokines, they also can favor tumor growth by Th2 cytokines (summarized in [95]). Interestingly, CD4 ${ }^{+} \mathrm{NKT}$ cells accumulate in HCC and were demonstrated to produce more Th2 cytokines than CD4- NKT cells [96], potentially suppressing the antitumor effect. Nevertheless, the observed effects have not been consistently found in all patients (or models), and the specific tumor microenvironment influences the function of NKT cells in tumor immunity [95]. The stark species differences in hepatic NKT cells (i.e., between mice and men) further hamper the translatability of NKT cell functions from rodent models to human HCC [97]. Thus, at present, it is still controversial whether NKT cells ultimately have a favorable or unfavorable impact on the patients' prognosis, and further research is needed, when considering NKT cell-based immunotherapies in patients with HCC.

\section{NK cells}

In HCC, the main cytotoxic interactions seem to be mediated by $\mathrm{CD} 8^{+} \mathrm{T}$ cells and $\mathrm{CD} 57^{+} \mathrm{NK}$ cells $[20,98]$. NKT cells, DCs and KCs activate NK cells, while Tregs and HSCs prevent the activation of NK cells [99]. Reduced amounts of NK cells were found in HCC lesions [100]. However, the role of NK cells in HCC is only partially understood. NK cell activation represents a highly regulated process that is driven by many different components of the tumor environment. As such, not only abiotic components (hypoxia, metabolites, acidosis, cytokines and growth factors) influence hepatic NK cells but also biotic players including stromal cells, regulatory immune cells and 'normal' neighboring cells contribute to NK cell activation. Moreover, a whole plethora of chemokines might trigger or inhibit NK cell homing to the malignant lesion, since they are equipped with several chemokine receptors [101]. In addition, NK cells are not only influenced by the microenvironment but themselves shape the tumor microenvironment (nicely summarized in [102]). Therefore, additional research is needed before a therapeutic use of NK cells to prevent hepatocarcinogenesis can be considered.

\section{Other cells in the tumor microenvironment}

Finally, nonparenchymal cells also modulate immune responses in HCC. For instance, HSCs inhibit T-cell activation by inducing MDSC [103] and by recruiting Tregs [104]. Liver sinusoidal endothelial cells activate Tregs via the TGF- $\beta$, inducing an immunosuppressive liver environment [105]. The role of so-called tumor-associated fibroblasts is not so well defined in HCC. Tumor-associated fibroblasts can inhibit antitumor response by impairing the function of NK cells and by facilitating MDSC generation in the tumor microenvironment [106]. 


\section{Conclusion}

The adaptive and innate immune system represents highly specialized but closely interconnected systems that maintain the integrity of the liver and protect it against external threats. In chronic liver diseases, innate immune mechanisms critically contribute to persistent inflammation, fibrosis and cirrhosis, thereby providing the seeding ground for hepatocarcinogenesis. In malignant diseases, innate and adaptive immunity are part of a highly differentiated tumor microenvironment, which can have both pro- and anti-carcinogenic effects and thus contribute decisively to the effectiveness of antitumor therapy. In this context, influencing the immune system and thereby shaping the tumor microenvironment toward the anticancerogenic side might help to control tumor growth and to improve patients' prognosis.

\section{Future perspective}

Current immunotherapies mainly rely on the adaptive arm of the immune system. A better understanding of the innate arm of the immune system might help to develop novel concepts with improved efficacy. Targeting innate immune mechanisms has the potential to improve immunosurveillance in inflamed liver, reduce HCC development in liver cirrhosis and boost conventional immune checkpoint inhibitor strategies in established HCC. Such novel concepts might help to 'find the holy grail' in immunooncology, namely, to turn immunologically 'cold' tumors 'hot.' At present, combinations of anti-PD-L1-/anti-PD-1-directed therapies are tested in numerous clinical trials. For instance, inhibiting the VEGFR pathway was shown not only to reprogram the tumor microenvironment but also to increase efficient priming and activation of T-cell responses enhancing response to anti-PD-L1/anti-PD-1 antibodies. In line, the IMBRAVE-150 trial that analyzed atezolizumab plus bevacizumab in patients with advanced HCC revealed a significantly higher efficacy of this combination compared with sorafenib. Moreover, different trials are investigating the role of MEK inhibition in the context of immunoncology, since MEK inhibition potentially increases recruitment of cytotoxic $\mathrm{T}$ cells into the tumor core, potentially reversing T-cell exhaustion. Finally, measures to increase contact of immune cells toward tumor antigens (radiation therapy, ablation, embolization) are analyzed in combination with anti-PD-L1/anti-PD-1 antibodies within large Phase III trials.

\section{Summary points}

- Hepatocellular carcinoma (HCC) is the fifth most common cancer worldwide and is still associated to a dismal prognosis.

- HCC represents an inflammation-driven disease and immune-oncological approaches are likely to improve patients' prognosis. However, recent Phase III trials have failed to demonstrate a survival benefit of anti-PD-1/PD-L1-directed therapies.

- In recent years, tumor-infiltrating immune cells have been extensively analyzed and characterized. Associations between distinct immune cell populations and response to therapy as well as on prognosis have been proposed in patients with HCC.

- The liver comprises a large population of innate immune cells in the body such as resident macrophages (Kupffer cells), natural killer cells or natural killer T cells, highlighting the important roles of these cells in the liver and in hepatocarcinogenesis.

- Influencing the innate immune system and thereby shaping the tumor microenvironment toward anticancerogenic actions might help to control tumor growth and to improve patients' prognosis.

- Combination therapies are tested in numerous clinical trials and most likely represent the future of immuno-oncology in HCC.

\section{Author contributions}

All the authors were involved in writing, editing and drafting the manuscript. All the authors approved the final version of the manuscript.

\section{Financial \& competing interests disclosure}

This work was funded by German Research Foundation (TA434/3-1, CRC1382). F Tacke's group has received research funding from Allergan, Inventiva, Bristol Myers Squibb and Galapagos. The other authors state no conflict of interest. The authors have no other relevant affiliations or financial involvement with any organization or entity with a financial interest in or financial conflict with the subject matter or materials discussed in the manuscript apart from those disclosed.

No writing assistance was utilized in the production of this manuscript. 
Open access

This work is licensed under the Attribution-NonCommercial-NoDerivatives 4.0 Unported License. To view a copy of this license, visit http://creativecommons.org/licenses/by-nc-nd/4.0/

\section{References}

Papers of special note have been highlighted as: $\bullet$ of interest

1. Torre LA, Bray F, Siegel RL, Ferlay J, Lortet-Tieulent J, Jemal A. Global cancer statistics, 2012. CA Cancer J. Clin. 65(2), 87-108 (2015).

- Important study providing information on the epidemiology of hepatocellular carcinoma (HCC).

2. GBD 2013 Mortality and Causes of Death Collaborators. Global, regional, and national age-sex specific all-cause and cause-specific mortality for 240 causes of death, 1990-2013: a systematic analysis for the Global Burden of Disease Study 2013. Lancet 385(9963), 117-171 (2015).

3. Higashi T, Friedman SL, Hoshida Y. Hepatic stellate cells as key target in liver fibrosis. Adv. Drug Deliv. Rev. 121, 27-42 (2017).

4. Mokdad AH, Dwyer-Lindgren L, Fitzmaurice C et al. Trends and patterns of disparities in cancer mortality among US counties, 1980-2014. JAMA 317(4), 388-406 (2017).

5. Bruix J, Takayama T, Mazzaferro V et al. Adjuvant sorafenib for hepatocellular carcinoma after resection or ablation (STORM): a Phase III, randomised, double-blind, placebo-controlled trial. Lancet Oncol. 16(13), 1344-1354 (2015).

6. Llovet JM, Ricci S, Mazzaferro V et al. Sorafenib in advanced hepatocellular carcinoma. N. Engl. J. Med. 359(4), 378-390 (2008).

7. Kudo M, Finn RS, Qin S et al. Lenvatinib versus sorafenib in first-line treatment of patients with unresectable hepatocellular carcinoma: a randomised Phase III non-inferiority trial. Lancet 391(10126), 1163-1173 (2018).

8. Bruix J, Qin S, Merle P et al. Regorafenib for patients with hepatocellular carcinoma who progressed on sorafenib treatment (RESORCE): a randomised, double-blind, placebo-controlled, Phase III trial. Lancet 389(10064), 56-66 (2017).

9. Abou-Alfa GK, Meyer T, Cheng AL et al. Cabozantinib in patients with advanced and progressing hepatocellular carcinoma. N. Engl. J. Med. 379(1), 54-63 (2018).

10. Parikh ND, Singal AG, Hutton DW. Cost effectiveness of regorafenib as second-line therapy for patients with advanced hepatocellular carcinoma. Cancer 123(19), 3725-3731 (2017).

11. Grivennikov SI, Greten FR, Karin M. Immunity, inflammation, and cancer. Cell 140(6), 883-899 (2010).

12. Mantovani A, Allavena P, Sica A, Balkwill F. Cancer-related inflammation. Nature 454(7203), 436-444 (2008).

13. El-Serag HB, Rudolph KL. Hepatocellular carcinoma: epidemiology and molecular carcinogenesis. Gastroenterology 132(7), $2557-2576$ (2007).

14. Ehling J, Tacke F. Role of chemokine pathways in hepatobiliary cancer. Cancer Lett. 379(2), 173-183 (2016).

15. European Association for the Study of the Liver, Galle PR, Forner A et al. EASL clinical practice guidelines: management of hepatocellular carcinoma. J. Hepatol. 69(1), 182-236 (2018).

16. Heimbach JK, Kulik LM, Finn RS et al. AASLD guidelines for the treatment of hepatocellular carcinoma. Hepatology 67(1), 358-380 (2018).

17. Kather JN, Halama N. Harnessing the innate immune system and local immunological microenvironment to treat colorectal cancer. $B r$. J. Cancer 120(9), 871-882 (2019).

18. El-Khoueiry AB, Sangro B, Yau T et al. Nivolumab in patients with advanced hepatocellular carcinoma (CheckMate 040): an open-label, non-comparative, Phase I/II dose escalation and expansion trial. Lancet 389(10088), 2492-2502 (2017).

19. Zhu AX, Finn RS, Edeline J et al. Pembrolizumab in patients with advanced hepatocellular carcinoma previously treated with sorafenib (KEYNOTE-224): a non-randomised, open-label Phase II trial. Lancet. Oncol. 19(7), 940-952 (2018).

20. Ringelhan M, Pfister D, O’Connor T, Pikarsky E, Heikenwalder M. The immunology of hepatocellular carcinoma. Nat. Immunol. 19(3), 222-232 (2018).

21. Rai V, Abdo J, Alsuwaidan AN, Agrawal S, Sharma P, Agrawal DK. Cellular and molecular targets for the immunotherapy of hepatocellular carcinoma. Mol. Cell. Biochem. 437(1-2), 13-36 (2018).

22. Zhang Q, He Y, Luo N et al. Landscape and dynamics of single immune cells in hepatocellular carcinoma. Cell 179(4), 829-845.e820 (2019).

- Using single-cell RNA sequencing from human HCC and adjacent liver tissue samples, this paper provides novel insights into the immune environment of HCC including functionally relevant inflammatory signatures of myeloid cells.

23. Ramachandran P, Dobie R, Wilson-Kanamori JR et al. Resolving the fibrotic niche of human liver cirrhosis at single-cell level. Nature 575(7783), 512-518 (2019).

24. Neureiter D, Stintzing S, Kiesslich T, Ocker M. Hepatocellular carcinoma: therapeutic advances in signaling, epigenetic and immune targets. World. J. Gastroenterol. 25(25), 3136-3150 (2019). 
25. Cariani E, Missale G. Immune landscape of hepatocellular carcinoma microenvironment: implications for prognosis and therapeutic applications. Liver Int. 39(9), 1608-1621 (2019).

26. Longo V, Gnoni A, Casadei Gardini A et al. Immunotherapeutic approaches for hepatocellular carcinoma. Oncotarget 8(20), 33897-33910 (2017).

27. Kudo M. Immune checkpoint inhibition in hepatocellular carcinoma: basics and ongoing clinical trials. Oncology 92(Suppl. 1), 50-62 (2017).

28. Kim TK, Herbst RS, Chen L. Defining and understanding adaptive resistance in cancer immunotherapy. Trends Immunol. 39(8), 624-631 (2018).

- Delineates mechanisms leading to resistance against immunotherapy in cancer.

29. Kather JN, Halama N, Jaeger D. Genomics and emerging biomarkers for immunotherapy of colorectal cancer. Semin. Cancer Biol. 52(Pt 2), 189-197 (2018).

30. Mossanen JC, Tacke F. Role of lymphocytes in liver cancer. Oncoimmunology 2(11), e26468 (2013).

31. Arlauckas SP, Garris CS, Kohler RH et al. In vivo imaging reveals a tumor-associated macrophage-mediated resistance pathway in anti-PD-1 therapy. Sci. Trans. Med. 9(389) (2017).

32. Zhou SL, Zhou ZJ, Hu ZQ et al. Tumor-associated neutrophils recruit macrophages and T-regulatory cells to promote progression of hepatocellular carcinoma and resistance to sorafenib. Gastroenterology 150(7), 1646-1658.e1617 (2016).

33. Cheng Y, Li H, Deng Y et al. Cancer-associated fibroblasts induce PDL1+ neutrophils through the IL6-STAT3 pathway that foster immune suppression in hepatocellular carcinoma. Cell Death Dis. 9(4), 422 (2018).

34. Benson DM Jr, Bakan CE, Mishra A et al. The PD-1/PD-L1 axis modulates the natural killer cell versus multiple myeloma effect: a therapeutic target for CT-011, a novel monoclonal anti-PD-1 antibody. Blood 116(13), 2286-2294 (2010).

35. Chen Z, Yang Y, Liu LL, Lundqvist A. Strategies to augment natural killer (NK) cell activity against solid tumors. Cancers 11(7) pii: E1040 (2019).

36. Turnis ME, Andrews LP, Vignali DA. Inhibitory receptors as targets for cancer immunotherapy. Eur. J. Immunol. 45(7), 1892-1905 (2015).

37. Wang J, Li Z, Gao A, Wen Q, Sun Y. The prognostic landscape of tumor-infiltrating immune cells in cervical cancer. Biomed. Pharmacother. 120, 109444 (2019).

38. Lam JH, Ng HHM, Lim CJ et al. Expression of CD38 on macrophages predicts improved prognosis in hepatocellular carcinoma. Front. Immunol. 10, 2093 (2019).

39. Chen F, Yang Y, Zhao Y, Pei L, Yan H. Immune infiltration profiling in nonsmall cell lung cancer and their clinical significance: study based on gene expression measurements. DNA Cell Biol. 38(11), 1387-1401 (2019).

40. Wan S, Kuo N, Kryczek I, Zou W, Welling TH. Myeloid cells in hepatocellular carcinoma. Hepatology 62(4), 1304-1312 (2015).

41. Barnes TA, Amir E. HYPE or HOPE: the prognostic value of infiltrating immune cells in cancer. Br. J. Cancer 117(4), 451-460 (2017).

42. Binnewies M, Roberts EW, Kersten $\mathrm{K}$ et al. Understanding the tumor immune microenvironment (TIME) for effective therapy. Nat. Med. 24(5), 541-550 (2018).

43. Anstee QM, Reeves HL, Kotsiliti E, Govaere O, Heikenwalder M. From NASH to HCC: current concepts and future challenges. Nat. Rev. Gastroenterol. Hepatol. 16(7), 411-428 (2019).

44. Ritz T, Krenkel O, Tacke F. Dynamic plasticity of macrophage functions in diseased liver. Cell. Immunol. 330, 175-182 (2018).

45. Ramachandran P, Dobie R, Wilson-Kanamori JR et al. Resolving the fibrotic niche of human liver cirrhosis at single-cell level. Nature 575(7783), 512-518 (2019).

46. Krenkel O, Hundertmark J, Abdallah AT et al. Myeloid cells in liver and bone marrow acquire a functionally distinct inflammatory phenotype during obesity-related steatohepatitis. Gut 69(3), 551-563 (2019).

47. Xiong X, Kuang H, Ansari S et al. Landscape of intercellular crosstalk in healthy and NASH liver revealed by single-cell secretome gene analysis. Mol. Cell 75(3), 644-660.e645 (2019).

48. Krenkel O, Hundertmark J, Ritz TP, Weiskirchen R, Tacke F. Single cell RNA sequencing identifies subsets of hepatic stellate cells and myofibroblasts in liver fibrosis. Cells 8(5) pii: E503 (2019).

49. Schneider C, Tacke F. Distinct anti-tumoral functions of adaptive immune cells in liver cancer. Oncoimmunology 1(6), 937-939 (2012).

50. Schneider C, Teufel A, Yevsa $\mathrm{T}$ et al. Adaptive immunity suppresses formation and progression of diethylnitrosamine-induced liver cancer. Gut 61(12), 1733-1743 (2012).

51. Krenkel O, Puengel T, Govaere $\mathrm{O}$ et al. Therapeutic inhibition of inflammatory monocyte recruitment reduces steatohepatitis and liver fibrosis. Hepatology 67(4), 1270-1283 (2018).

52. Eggert T, Wolter K, Ji J et al. Distinct functions of senescence-associated immune responses in liver tumor surveillance and tumor progression. Cancer Cell 30(4), 533-547 (2016).

- Fundamental paper describing two opposite roles of macrophages in liver cancer initiation and tumor control. 
53. Wolf MJ, Adili A, Piotrowitz K et al. Metabolic activation of intrahepatic CD8+ T cells and NKT cells causes nonalcoholic steatohepatitis and liver cancer via cross-talk with hepatocytes. Cancer Cell 26(4), 549-564 (2014).

- Delineates mechanisms leading to resistance against immunotherapy in cancer.

54. Li X, Yao W, Yuan Y et al. Targeting of tumour-infiltrating macrophages via CCL2/CCR2 signalling as a therapeutic strategy against hepatocellular carcinoma. Gut 66(1), 157-167 (2017).

55. Bartneck M, Schrammen PL, Mockel D et al. The CCR2(+) macrophage subset promotes pathogenic angiogenesis for tumor vascularization in fibrotic livers. Cell. Mol. Gastroenterol. Hepatol. 7(2), 371-390 (2019).

56. Yao W, Ba Q, Li X et al. A natural CCR2 antagonist relieves tumor-associated macrophage-mediated immunosuppression to produce a therapeutic effect for liver cancer. EBioMedicine 22, 58-67 (2017).

57. Tacke F. Cenicriviroc for the treatment of non-alcoholic steatohepatitis and liver fibrosis. Expert. Opin. Investig. Drugs 27(3), 301-311 (2018).

58. Friedman SL, Ratziu V, Harrison SA et al. A randomized, placebo-controlled trial of cenicriviroc for treatment of nonalcoholic steatohepatitis with fibrosis. Hepatology 67(5), 1754-1767 (2018).

59. Maeda S, Kamata H, Luo JL, Leffert H, Karin M. IKKbeta couples hepatocyte death to cytokine-driven compensatory proliferation that promotes chemical hepatocarcinogenesis. Cell 121(7), 977-990 (2005).

60. Koh MY, Gagea M, Sargis T et al. A new HIF-1alpha/RANTES-driven pathway to hepatocellular carcinoma mediated by germline haploinsufficiency of SART1/HAF in mice. Hepatology 63(5), 1576-1591 (2016).

61. Malehmir M, Pfister D, Gallage $\mathrm{S}$ et al. Platelet GPIbalpha is a mediator and potential interventional target for NASH and subsequent liver cancer. Nat. Med. 25(4), 641-655 (2019).

62. Yuan D, Huang S, Berger E et al. Kupffer cell-derived Tnf triggers cholangiocellular tumorigenesis through JNK due to chronic mitochondrial dysfunction and ROS. Cancer Cell 31(6), 771-789.e776 (2017).

63. Krenkel O, Tacke F. Liver macrophages in tissue homeostasis and disease. Nat. Rev. Immunol. 17(5), 306-321 (2017).

64. Robert O, Boujedidi H, Bigorgne A et al. Decreased expression of the glucocorticoid receptor-GILZ pathway in Kupffer cells promotes liver inflammation in obese mice. J. Hepatol. 64(4), 916-924 (2016).

65. Fan Z, Li L, Li M et al. The histone methyltransferase Suv39h2 contributes to nonalcoholic steatohepatitis in mice. Hepatology 65(6), 1904-1919 (2017).

66. Szabo G, Petrasek J. Inflammasome activation and function in liver disease. Nat. Rev. Gastroenterol. Hepatol. 12(7), 387-400 (2015).

67. Kong L, Zhou Y, Bu H, Lv T, Shi Y, Yang J. Deletion of interleukin-6 in monocytes/macrophages suppresses the initiation of hepatocellular carcinoma in mice. J. Exp. Clin. Cancer Res. 35(1), 131 (2016).

68. Sun K, Xu L, Jing Y et al. Autophagy-deficient Kupffer cells promote tumorigenesis by enhancing mtROS-NF-kappaB-IL1alpha/beta-dependent inflammation and fibrosis during the preneoplastic stage of hepatocarcinogenesis. Cancer Lett. 388, 198-207 (2017).

69. Kessoku T, Imajo K, Honda Y et al. Resveratrol ameliorates fibrosis and inflammation in a mouse model of nonalcoholic steatohepatitis. Sci. Rep. 6, 22251 (2016).

70. Wagner W, Wein F, Roderburg C et al. Adhesion of human hematopoietic progenitor cells to mesenchymal stromal cells involves CD44. Cells, Tissues, Organs 188(1-2), 160-169 (2008).

71. Medina-Echeverz J, Eggert T, Han M, Greten TF. Hepatic myeloid-derived suppressor cells in cancer. Cancer Immunol. Immunother. 64(8), 931-940 (2015).

72. Talmadge JE, Gabrilovich DI. History of myeloid-derived suppressor cells. Nat. Rev. Cancer 13(10), 739-752 (2013).

73. Oleinika K, Nibbs RJ, Graham GJ, Fraser AR. Suppression, subversion and escape: the role of regulatory T cells in cancer progression. Clin. Exper. Immunol. 171(1), 36-45 (2013).

74. Ouyang FZ, Wu RQ, Wei Y et al. Dendritic cell-elicited B-cell activation fosters immune privilege via IL-10 signals in hepatocellular carcinoma. Nat. Commun. 7, 13453 (2016).

75. Wiedemann GM, Knott MM, Vetter VK et al. Cancer cell-derived IL-1alpha induces CCL22 and the recruitment of regulatory T cells. Oncoimmunology 5(9), e1175794 (2016).

76. Yu SJ, Ma C, Heinrich B et al. Targeting the crosstalk between cytokine-induced killer cells and myeloid-derived suppressor cells in hepatocellular carcinoma. J. Hepatol. 70(3), 449-457 (2019).

77. Hsieh CC, Hung CH, Chiang M, Tsai YC, He JT. Hepatic stellate cells enhance liver cancer progression by inducing myeloid-derived suppressor cells through interleukin-6 signaling. Int. J. Mol. Sci. 20(20) (2019).

78. Liu M, Zhou J, Liu X et al. Targeting monocyte-intrinsic enhancer reprogramming improves immunotherapy efficacy in hepatocellular carcinoma. Gut 69(2), 365-379 (2019).

79. Galdiero MR, Bonavita E, Barajon I, Garlanda C, Mantovani A, Jaillon S. Tumor associated macrophages and neutrophils in cancer. Immunobiology 218(11), 1402-1410 (2013). 
80. Dumitru CA, Lang S, Brandau S. Modulation of neutrophil granulocytes in the tumor microenvironment: mechanisms and consequences for tumor progression. Semin. Cancer Biol. 23(3), 141-148 (2013).

81. Li X, Xing YF, Lei AH et al. Neutrophil count is associated with myeloid derived suppressor cell level and presents prognostic value of for hepatocellular carcinoma patients. Oncotarget 8(15), 24380-24388 (2017).

82. Houghton AM, Rzymkiewicz DM, Ji H et al. Neutrophil elastase-mediated degradation of IRS-1 accelerates lung tumor growth. Nat. Med. 16(2), 219-223 (2010).

83. Spicer JD, McDonald B, Cools-Lartigue JJ et al. Neutrophils promote liver metastasis via Mac-1-mediated interactions with circulating tumor cells. Cancer Res. 72(16), 3919-3927 (2012).

84. Huh SJ, Liang S, Sharma A, Dong C, Robertson GP. Transiently entrapped circulating tumor cells interact with neutrophils to facilitate lung metastasis development. Cancer Res. 70(14), 6071-6082 (2010).

85. Ardi VC, Kupriyanova TA, Deryugina EI, Quigley JP. Human neutrophils uniquely release TIMP-free MMP-9 to provide a potent catalytic stimulator of angiogenesis. Proc. Natl Acad. Sci. USA 104(51), 20262-20267 (2007).

86. Wang TT, Zhao YL, Peng LS et al. Tumour-activated neutrophils in gastric cancer foster immune suppression and disease progression through GM-CSF-PD-L1 pathway. Gut 66(11), 1900-1911 (2017).

87. Xu R, Huang H, Zhang Z, Wang FS. The role of neutrophils in the development of liver diseases. Cell Mol. Immunol. 11(3), 224-231 (2014).

88. Wehr A, Baeck C, Heymann F et al. Chemokine receptor CXCR6-dependent hepatic NK T Cell accumulation promotes inflammation and liver fibrosis. J. Immunol. 190(10), 5226-5236 (2013).

89. Liepelt A, Wehr A, Kohlhepp M et al. CXCR6 protects from inflammation and fibrosis in NEMO ${ }^{\text {LPC-KO }}$ mice. Biochim. Biophys. Acta, Mol. Basis Dis. 1865(2), 391-402 (2019).

90. Tahir SM, Cheng O, Shaulov A et al. Loss of IFN-gamma production by invariant NK T cells in advanced cancer. J. Immunol. 167(7), 4046-4050 (2001).

91. Mossanen JC, Kohlhepp M, Wehr A et al. CXCR6 inhibits hepatocarcinogenesis by promoting natural killer T- and CD4(+) T-cell-dependent control of senescence. Gastroenterology 156(6), 1877-1889.e1874 (2019).

- Links specific lymphocyte populations to the immunosurveillance of senescent hepatocytes during carcinogenesis in the liver.

92. Ma C, Han M, Heinrich B et al. Gut microbiome-mediated bile acid metabolism regulates liver cancer via NKT cells. Science 360(6391) (2018).

93. Syn WK, Agboola KM, Swiderska M et al. NKT-associated hedgehog and osteopontin drive fibrogenesis in non-alcoholic fatty liver disease. Gut 61(9), 1323-1329 (2012).

94. Syn WK, Oo YH, Pereira TA et al. Accumulation of natural killer T cells in progressive nonalcoholic fatty liver disease. Hepatology 51(6), 1998-2007 (2010).

95. Zhu S, Zhang H, Bai L. NKT cells in liver diseases. Front. Med. 12(3), 249-261 (2018).

96. Bricard G, Cesson V, Devevre E et al. Enrichment of human CD4+ V(alpha)24/Vbeta11 invariant NKT cells in intrahepatic malignant tumors. J. Immunol. 182(8), 5140-5151 (2009).

97. Heymann F, Tacke F. Immunology in the liver - from homeostasis to disease. Nat. Rev. Gastroenterol. Hepatol. 13(2), 88-110 (2016).

- Comprehensive review on immunology in the liver and in liver diseases.

98. Garnelo M, Tan A, Her Z et al. Interaction between tumour-infiltrating B cells and T cells controls the progression of hepatocellular carcinoma. Gut 66(2), 342-351 (2017).

99. Tian Z, Chen Y, Gao B. Natural killer cells in liver disease. Hepatology 57(4), 1654-1662 (2013).

100. Cai L, Zhang Z, Zhou L et al. Functional impairment in circulating and intrahepatic NK cells and relative mechanism in hepatocellular carcinoma patients. Clin. Immunol. 129(3), 428-437 (2008).

101. Marra F, Tacke F. Roles for chemokines in liver disease. Gastroenterology 147(3), 577-594.e571 (2014).

102. Pineiro Fernandez J, Luddy KA, Harmon C, O'Farrelly C. Hepatic tumor microenvironments and effects on NK cell phenotype and function. Int. J. Mol. Sci. 20(17) pii: E4131 (2019).

103. Hochst B, Schildberg FA, Sauerborn P et al. Activated human hepatic stellate cells induce myeloid derived suppressor cells from peripheral blood monocytes in a CD44-dependent fashion. J. Hepatol. 59(3), 528-535 (2013).

104. Dunham RM, Thapa M, Velazquez VM et al. Hepatic stellate cells preferentially induce Foxp3+ regulatory T cells by production of retinoic acid. J. Immunol. 190(5), 2009-2016 (2013).

105. Carambia A, Freund B, Schwinge D et al. TGF-beta-dependent induction of CD4(+)CD25(+)Foxp3(+) Tregs by liver sinusoidal endothelial cells. J. Hepatol. 61(3), 594-599 (2014).

106. Deng Y, Cheng J, Fu B et al. Hepatic carcinoma-associated fibroblasts enhance immune suppression by facilitating the generation of myeloid-derived suppressor cells. Oncogene 36(8), 1090-1101 (2017). 